\title{
Praxisneuling
}

\section{Ist das in Ordnung: Plausibilitätsprüfung gleich bei der ersten Abrechnung?}

Frage von Dr. med. N. N.:

Ich habe vor vier Jahren eine Allgemeinarztpraxis übernommen. Im ersten Quartal war die Situation neu, und es gab eine Grippewelle, sodass ich viel Zeit in der Praxis verbracht habe. Vier Jahre später wird eine Plausibilitätsprüfung seitens der KV durchgeführt und mir eine Strafe auferlegt. Es sei zu viel abgerechnet worden, so viel könne man täglich nicht arbeiten.

Tatsächlich hat mich damals die Tätigkeit sehr überfordert, sodass ich bis zu zwölf Stunden täglich in der Praxis verbracht habe. Darf man die erste Abrechnung eines niedergelassenen Arztes bestrafen, ohne dass eine vorherige $\mathrm{Be}$ ratung durch die KV erfolgt ist?

\section{Antwort von RA M. Broglie:}

Plausibilitätsprüfungen sind immer möglich. Die kritischen Zeiten stellen nur Aufgreifkriterien dar. Mit längeren Arbeitszeiten kann man durchaus die Plausibilität wieder herstellen. Jeder Arzt sollte aber mit Hilfe seiner Praxis- software ständig die eigene Plausibilitätsprüfung vornehmen, um vor Überraschungen sicher zu sein. Nicht selten kann so eine Plausibilitätsprüfung auch $\mathrm{zu}$ erheblichen Rückzahlungen an die $\mathrm{KV}$ oder zu strafrechtlichen Konsequenzen führen. Unter Umständen ist die Hinzuziehung eines im Medizinrecht und im ärztlichen Honorarwesen erfahrenen Anwaltes ratsam.

- Rechtsanwalt Maximilian Guido Broglie, Fachanwalt für Medizin- und Sozialrecht, Sonnenberger Straße 16, D-65193 Wiesbaden

\section{Wird von Personal Trainern empfohlen}

\section{Wozu ist Betainhydrochlorid gut?}

Frage von Dr. med. B. H.:

Zur Zeit höre ich immer wieder von $\mathrm{Pa}$ tienten, dass ihnen ihr „Personal Trainer“ im Rahmen einer eiweißbetonten Ernährung $\mathrm{HCl}$ Betain (Betainhydrochlorid) empfohlen habe. Dies wird offenbar des Öfteren nach einem „Test“ auf Hypo-chlorhydrie angeraten. Ich kenne hierzu keine valide Datenlage.

Mich würde vor allem interessieren, ob es für eine solche Supplementation eine Indikation gibt und wie diese begründet wird. Auch wüsste ich gerne, ob die Supplementation in der Schwangerschaft und Stillzeit mit Risiken verbunden ist.

\section{Antwort von Apothekerin A.}

Rolfes-Bußmann und Prof. Dr. med.

\section{J. C. Frölich:}

Als Nahrungsergänzungsmittel ist Betainhydrochlorid zur HCl-Substitution bei anacidem Magen im Handel. Eine effektive Therapie ist dabei nicht zu erwarten bzw. nicht erforderlich, da die Verdau- ung in der Regel durch die Pankreasenzyme gesichert ist. Für andere Betainsalze sind im Tierversuch hepatoprotektive Eigenschaften gezeigt worden.

Betain ist als Arzneimittel zur Behandlung der Homocystinurie zugelassen. Als Methylgruppendonator bewirkt es die Remethylierung von Homocystein zu Methionin, wodurch die Homo-

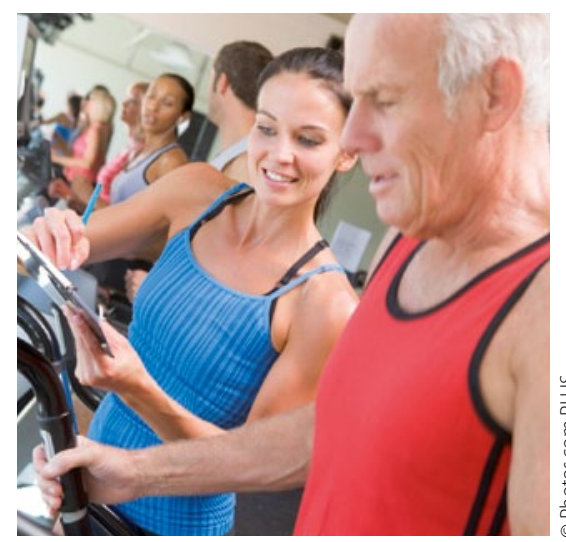

In Fitnessstudios gibt es auch (fragwürdige?) Ernährungstipps.
cystein-Spiegel sinken. Zur Anwendung in der Schwangerschaft ist in der Fachinformation von Cystadane ${ }^{\circledast} \mathrm{zu}$ lesen: „Strenge Indikationsstellung. Daten über eine begrenzte Anzahl von exponierten Schwangeren lassen nicht auf Nebenwirkungen von Betain auf die Schwangere oder die Gesundheit des Fetus/Neugeborenen schließen. Während der Schwangerschaft sollte eine Anwendung von Betain [...] unter engmaschiger Kontrolle des Homocysteinspiegels im Plasma vereinbar mit guten Ergebnissen sowohl für die Mutter als auch das ungeborene Kind sein.“

Eine Supplementierung mit Betainhydrochlorid ist bei ausgewogener Ernährung in der Regel nicht notwendig und sollte erst nach ärztlicher Diagnosestellung erfolgen, insbesondere bei Schwangeren.

- A. Rolfes-Bußmann, Apothekerin, Prof. Dr. med. J. C. Frölich, Institut für Klinische Pharmakologie, Medizinische Hochschule Hannover, D-30623 Hannover 